\title{
HYPERTROPHY OF THE PALMARIS LONGUS MUSCLE
}

\section{Report of a Case}

\section{B. Sterry Ashib, London, England}

A girl of thirteen attended St James's Hospital, Balham, with a swelling on the front of the right forearm. She complained of a tingling sensation in the thumb, the index, middle and ring fingers and up the forearm, and had aching and weakness in the wrist and hand when she was tired. The symptoms had been present for three years. She was left handed.

On examination a swelling six centimetres long was situated in the mid-line of the flexor aspect in the lowest third of the right forearm. The swelling had a faint bluish tinge but it was not tender or cystic. The radial pulse was normal. There was no sensory loss, no motor weakness or muscle wasting in the hand and she had a good grip. A normal palmaris longus tendon was palpated on the left side.

Both hands showed a " simian" configuration with a coarse skin and deep skin lines (Fig. 1). The feet also were wrinkled with deep skin lines and she had coarse scaly skin over the trunk and legs. She was one of a family of twelve children; her mother had deep skin lines on her hands and feet and her younger brother aged eleven had similar hands and feet and coarse skin on his body.

At operation a three-centimetre transverse incision was made over the distal part of the swelling. A large fleshy muscle belly was found in place of the palmaris longus tendon (Fig. 2). The position and appearance of the median nerve was normal. The muscle was detached from the flexor retinaculum and pulled through a second transverse incision higher up the forearm and divided as high up as possible. Histological examination showed normal muscle tissue. The operation completely relieved the patient's symptoms.

\section{DISCUSSION}

The long digital flexor muscles in the forearm are arranged in three layers. The flexor digitorum profundus (the deep layer) flexes the distal interphalangeal joints; the flexor digitorum sublimis (intermediate layer) flexes the proximal interphalangeal joints; and the palmaris longus (representing the superficial stratum), the primitive function of which is flexion of the metacarpo-phalangeal joints by means of a tendon that fans out in the palm to be inserted by a slip to the base of each proximal phalanx. In the development of the forelimb as a prehensile organ this function has been taken over by the intrinsic muscles of the hand and the palmaris longus muscle has become degenerate. The attenuated muscle belly is largely replaced by tendon and the degenerate radiating tendons of the palm have become the central portion of the palmar fascia, which retains the five distinct slips of attachment (Wood Jones 1941).

It is notable that this almost functionless muscle is present in about 90 per cent of humans. Degeneration has proceeded much further in the group of animals generally considered as phylogenetic forebears. Some remnant of it is usually present in the gibbon and orang-utan, but less often in the chimpanzee and ape, and it is only present in about 25 per cent of gorillas. In this respect the palmaris longus belongs to a group of muscles which are more degenerate in the apes and monkeys than in man. Others include the palmaris brevis muscle which is peculiar to man; and the plantaris, which is a primitive muscle retained in man but seldom in other primates, despite the specialised development of the foot as an organ of orthograde progression (Wood Jones 1941). 
It is well known that variations will occur in a structure which is undergoing retrogression, but it is uncommon for hypertrophy to occur. Reimann, Daseler, Anson and Beaton (1944), in a study of 1,600 limbs, found the palmaris longus muscle to be absent in 12.8 per cent but in 530 limbs they also found it present but abnormal in another 9 per cent (forty-six cases). In twenty-three of these there was a variation in the muscle, and in the other twenty-three the anomaly was an accessory slip or substitute structure. Duplication of the muscle occurred in four limbs. Reversal of the muscle, so that the belly lay distally, was not recorded and there were no examples of hypertrophy.

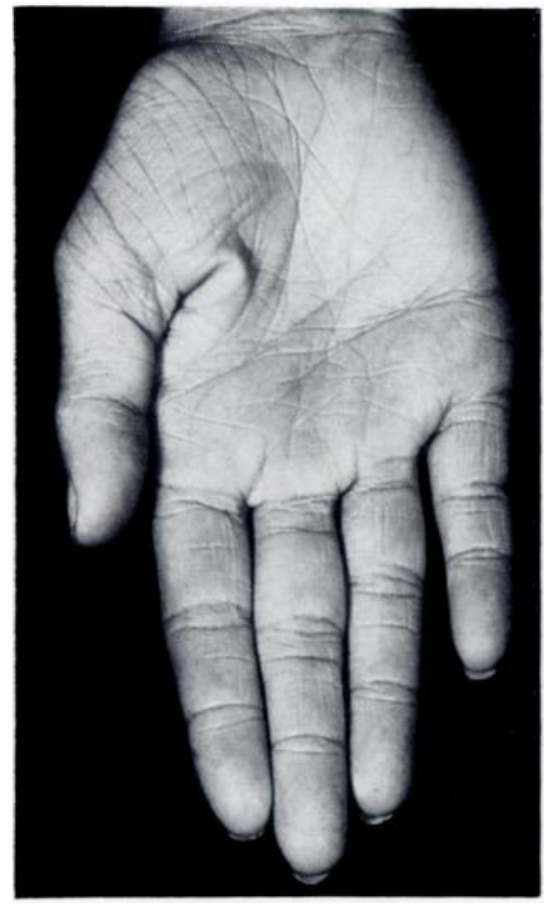

FiG. 1

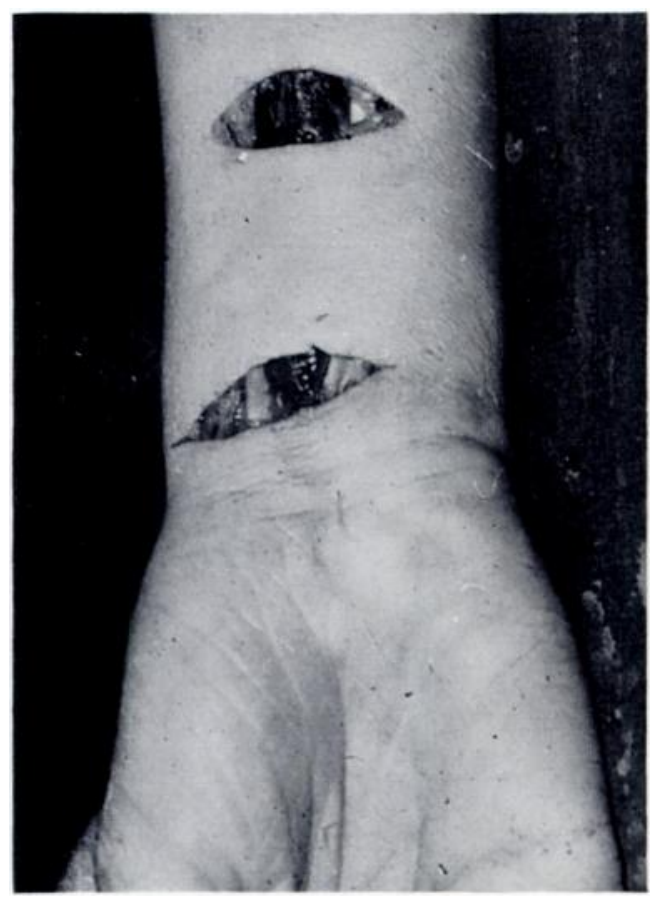

Fig. 2

Figure 1-The patient's right hand. Note the unusually coarse, deep-lined skin for a girl of thirteen. Figure 2-Exposure at operation. The fleshy muscle belly can be seen in place of the usual slim palmaris longus tendon.

It is evident therefore that although variations in the configuration of the palmaris longus muscle are fairly common, hypertrophy is rare and moreover unexpected in a structure which is retrogressing in practically all the species in which it still exists. In the case described in this paper the palmaris longus had developed into a hypertrophied muscle belly in place of the normal slim tendon and this was causing mild symptoms of median nerve compression which were completely relieved by removal of the abnormal muscle.

No account of an exactly comparable case has been found in the literature. Goulding (1948) described marked hypertrophy of the palmaris longus muscle in the right arm of a twenty-two-year-old man, in whom a dull aching pain accompanied the swelling. The muscle belly was excised and the symptoms were relieved. Madsen (1947) recorded two cases of an abnormal palmaris longus muscle, one of which showed a fleshy belly in place of the usual tendon.

The present patient showed other interesting features besides the unilateral hypertrophy of the palmaris longus muscle. Her hands and feet had coarse skin and deep lines, and the skin all over the legs and trunk was coarse and scaly. Her mother also had unusually coarse skin lines of the hands and feet, and her younger brother had similar hands and scaly skin all over his body. 


\section{SUMMARY}

A case of unilateral hypertrophy of the palmaris longus muscle in a girl of thirteen is described, associated with "simian" hands and feet and unusually coarse skin of the trunk. Symptoms of median nerve compression were relieved by excision of the muscle.

I would like to thank Mr A. M. Desmond, under whose care the patient was admitted to St James's Hospital, Balham, for permission and encouragement to publish this case report, and Miss E. Mason for the clinical photographs.

\section{REFERENCES}

Goulding, R. (1948): Gross Hypertrophy of the Palmaris Longus Muscle, Simulating a Tumour of the Forearm. British Journal of Surgery, 36, 213.

Jones, F. Wood (1941): The Principles of Anatomy as Seen in the Hand. Second edition. London: Baillière, Tindall and Cox.

Madsen, E. (1947): Musculus Palmaris Longus Inversus. Nordisk Medicin, 34, 855.

Reimann, A. F., Daseler, E. H., Anson, B. J., and Beaton, L. E. (1944): The Palmaris Longus Muscle and Tendon. A Study of 1,600 Extremities. Anatomical Record, 89, 495. 